\title{
Conformational analysis, energy profile, and structural-electronic properties evaluation of mephedrone derivatives employing quantum-mechanical models
}

\author{
Wojciech Kolodziejczyk ${ }^{1,2}$ (D) Supratik $\operatorname{Kar}^{2}$ - Glake A. Hill ${ }^{2}$
}

Received: 24 September 2016 / Accepted: 7 November 2016 / Published online: 19 November 2016

(C) The Author(s) 2016. This article is published with open access at Springerlink.com

\begin{abstract}
Mephedrone (4-methyl-meth-cathinone) or 4-MMC and its derivative 3-MMC are analogs of cathinone which act as non-selective substrates for monoamine transporters, facilitating a neurotransmitter release via the reversal of normal transporter fluxes. The pharmacological effects like paranoia, hallucinations, euphoria, aggressiveness, and psychosis make these molecules "abusive designer drugs." In the present study, conformations of all isomers of 3- and 4-MMC have been studied at the quantum-chemical level. Calculations have been performed using Møller-Plesset second-order (MP2) methods with 6$31 \mathrm{G}(\mathrm{d}, \mathrm{p})$ basis sets. Present study shows that there are lowenergy conformers for the meta (3-MMC) and para (4-MMC) isomers that are connected by way of low-barrier transition states. Natural bond orbital (NBO) analyses have been also performed to corroborate the results as estimated from the MP2 calculations. The molecular electrostatic potential surface data for each molecule has been calculated, revealing likely that reaction sites and probable metabolism pathway for both derivatives have been proposed. IR spectra for MMC derivatives have been compared with experimental data, and rotational transition states connecting all conformers have been reported.
\end{abstract}

Electronic supplementary material The online version of this article (doi:10.1007/s11224-016-0878-7) contains supplementary material, which is available to authorized users.

Wojciech Kolodziejczyk

dziecial@icnanotox.org

1 Department of Physical Chemistry, Wroclaw Medical University, ul. Borowska 211A, 50-556 Wroclaw, Poland

2 Interdisciplinary Center for Nanotoxicity, Jackson State University, Jackson, MS 39217, USA
Keywords Cathinone - Conformations - Gaussian · Mephedrone $\cdot$ Molecular electrostatic potential $\cdot$ MP2

\section{Introduction}

Cathinone ( $\beta$-keto-amphetamine), a naturally occurring psychoactive constituent obtained from the leaves of khat (Catha edulis), is a basis for the development of a series of synthetic cathinone derivatives used recreationally [1]. Designer cathinones easily permeate the blood-brain barrier (BBB) layer, eliciting sympathomimetic and psychostimulant actions by acting as a central nervous system stimulant by promoting the release of monoamine neurotransmitters and likely inhibiting their uptake. Their effects in humans include paranoia, hallucinations, euphoria, aggressiveness, psychosis, and an increment of libido, in addition to the more serious occurrences of hypertension, hyperthermia, seizures, respiratory distress, together with reports of fatal outcomes $[2,3]$. The higher number of intoxication reports during the last 10 years has led to laws that banned the use and sale of synthetic cathinones throughout different countries, although they continue to be commercialized worldwide illegally as components of illicit bath salts or legal highs [4]. Because of its amphetamine-like effects and its resultant high-abuse potential, cathinone derivatives were assigned schedule I status by the US Drug Enforcement Administration (DEA) in 1993 and class B status under the UK Misuse of Drugs Act in 1998 [5].

Mephedrone (4-methylmethcathinone)/4-MMC is parasubstituted meth-cathinone analog, one of the most commonly employed designer drug in "legal highs" drug moiety, most commonly available cathinone sold on recreational market in the period up to 2010 that was first time synthesized in 1929 [6]. 4-MMC was mainly used for illicit recreational consumption due to their psychoactive effects, which primarily act as substrate for monoamine transporters, facilitating the release 
of neurotransmitters via the reversal of normal transporter fluxes [2]. Of note, recent reports [7, 8] have demonstrated that 4-MMC display similar potencies to increase the release of monoamines in vitro, with a low selectivity for dopamine (DA) versus serotonin (5-HT) transporters (DAT and SERT, respectively). 4-MMC was widely used in East Africa and the Arab Peninsula as an amphetamine-like stimulant and popularized in Europe and the USA as one of the primary components of bath salts during the beginning of twenty-first century [9]. Because of its rapid rise to international notoriety as a designer drug of abuse, as well as its cocaine- and methamphetamine-like discriminative stimulus effects, 4MMC was added to the list of controlled drugs in both the USA and the UK in 2010-2011 [10].

Mephedrone has two major derivatives, 2-methyl methcathinone (2-MMC) and 3-methyl meth-cathinone (3-MMC) [11-13]. Due to structural similarity with 4-MMC, comparable pharmacological actions are noticed for both derivatives. Though, 4-MMC is controlled in the Republic of Ireland since May 2010 but 2-MMC and 3-MMC are not controlled. All derivatives of cathinone are controlled in Poland since 2010, but they are legal in Czech Republic. The banned 4-MMC leads to create more demand of 3-MMC in the "legal highs" market, and the major concern is the limited analytical data available about 3-MMC derivatives and their metabolites. These legal and analytical problems prompted us for quantum chemical analysis of 3-MMC along with major cathinone derivative 4-MMC molecule.

The primary aim of this study is to identify all possible conformers of 3- and 4-MMC (Fig. 1), so that these structures may be compared to cathinone structure. These derivatives are considered for $\mathrm{ab}$ initio treatments for energy profiling along with that molecular electrostatic potential surfaces (MEPSs) are also calculated. The natural bond orbital (NBO) analyses have been also performed to corroborate the results as estimated from the Møller-Plesset second-order (MP2) calculations. Additionally, the possible metabolism path has been also introspected.

\section{Computational methods and analysis}

The obtained results from our previous work [14] helped us to choose the best optimization method and basis set; therefore,<smiles>CNC(C)C(=O)c1ccc(C)cc1</smiles>

4-methylmethcathinone (4-MMC) full geometry optimization has been carried out using MP2 calculations [15] with 6-31G(d,p) basis sets employing Gaussian 09 package [16]. Potential energy surfaces were scanned, and all minima were optimized to find all possible conformers of 3-MMC and 4-MMC (mephedrone). The transition states have been obtained with one imaginary frequency. The molecular energy potential surface has been prepared using GaussView program.

The thermodynamic parameters of all compounds were calculated using statistical mechanic expressions utilizing the ideal gas, rigid rotor, and harmonic oscillator approximations. Solvation-free energies $\left(\Delta G_{\text {solv }}\right)$ were calculated at the MP2 level, using COSMO [17] in water solvent. The $\Delta G_{\text {solv }}$ values were calculated in aqueous continuum $(\varepsilon=78.39)$, and the molecular cavities were built using the united atom for the HartreeFock (UAHF) procedure, as used in COSMO. In principle, the free energy surfaces are determined by rigorous combination of free energy perturbation umbrella sampling approaches [18, 19]. These surfaces are very rigorous, as they reflect non-equilibrium solvation. However, such calculations are very challenging when performed within the ab initio framework. Thus, a more practical, simplified approach has been made. The relative free energies $(\Delta G)$ are determined as [20]

$$
\begin{aligned}
& \Delta G=\Delta H_{\mathrm{gas}}(298 \mathrm{~K})-T \Delta S-\mathrm{RT} \ln (\omega)+\Delta \Delta G_{\mathrm{solv}} \\
& \quad \approx \Delta G_{\mathrm{solv}}(298 \mathrm{~K})+\Delta \Delta G_{\mathrm{solv}}
\end{aligned}
$$

In Eq. (1), $\Delta H_{\text {gas }}(298 \mathrm{~K})$ is the enthalpy at $298 \mathrm{~K}$, $\Delta S$ is the gas-phase entropy, $\Delta G_{\text {gas }}(298 \mathrm{~K})$ is the Gibb's free energy at $298 \mathrm{~K}$, and $\Delta G_{\text {solv }}$ is the relative free energy of solvation. The contribution of the RT $\ln$ $(\omega)$ term is zero, as the electronic degeneracy term, $\omega$, for the singlet state is unity. The $\Delta G$ values are used to calculate the relative population of various conformers of the molecules, under study, in aqueous medium.

Since the molecules are much more floppy in solution than in the gas phase, we have used another definition of free energy $\left(\Delta g_{\text {flex }}\right)$ to compare the intrinsic flexibility of different conformers, when embedded in aqueous medium. This is the practical implementation of the more general expression used by Warshel and<smiles>CNC(C)C(=O)c1cccc(C)c1</smiles>

\section{3-methylmethcathinone (3-MMC)}

Fig. 1 Structures of 3-methylmethcathinone (3-MMC, meta) and 4-methylmethcathinone (4-MMC, para) 
Fig. 2 All six conformers of 3MMC calculated with $6-31 \mathrm{G}(\mathrm{d}, \mathrm{p})$ basis set on MP2 level

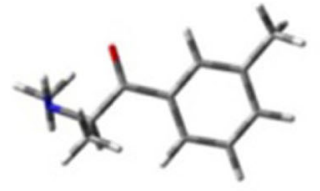

a

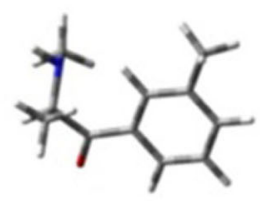

d

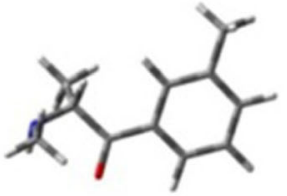

b

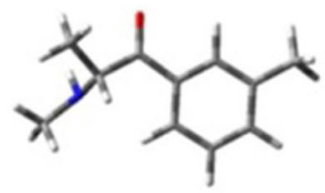

e

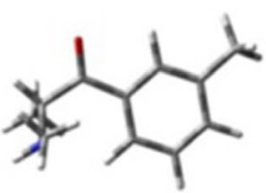

C

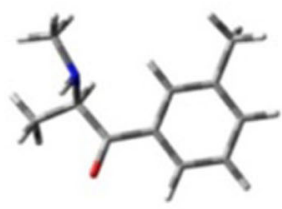

f coworkers $[20,21]$. The $\Delta g_{\text {flex }}$ could be expressed as

$\Delta g_{\text {flex }}=\Delta E_{\text {solute }}+\Delta \mathrm{ZPE}+\Delta \Delta G_{\text {solv }}-\alpha \mathrm{T} \Delta \mathrm{S}$

where $\Delta E_{\text {solute }}$ and $\Delta \mathrm{ZPE}$ in Eq. (2) are the relative gas-phase energy separations and the zero-point energies of the various conformers. The scale factor $\alpha$ is usually taken as zero [21], and Eq. (2) can be simplified as

$\psi_{\text {gflex }}=\Delta H_{0}^{\text {gas }}+\Delta \Delta G_{\text {solv }}$

All terms in Eqs. (1) and (3) are available from the thermochemical analyses based on statistical mechanic expressions using the ideal gas, rigid rotator, and harmonic oscillator approximations [22]. Population has been calculated using Boltzmann equation. The thermodynamic parameters of all compounds were calculated using statistical mechanic expressions utilizing the ideal gas, rigid rotor, and harmonic oscillator approximations.

The NBO analysis was generated using NBO 6.0 program [23]. NBO 6.0 is a critical detection program for studying complicated wave functions like interactions between bonds [24].

\section{Results and discussions}

\section{3-MMC}

All conformers of 3-MMC (Fig. 2) have been calculated using MP2 level of theory. Results show that 3-MMC has six
Table 1 Thermodynamic data of low-energy conformers of 3 $\mathrm{MMC}$ in $\mathrm{kcal} / \mathrm{mol}$ in gas and solution phase calculated at MP2 level

\begin{tabular}{|c|c|c|c|c|c|}
\hline Phase & Conformer & $\Delta H_{0}(\mathrm{kcal} / \mathrm{mol})$ & $\Delta G(\mathrm{kcal} / \mathrm{mol})$ & Dipole moment (D) & Population (\%) \\
\hline \multirow[t]{15}{*}{ Gas } & $a$ & 0.00 & 0.00 & 3.31 & 47.02 \\
\hline & $b$ & 1.53 & 1.29 & 2.51 & 3.55 \\
\hline & $c$ & 1.57 & 1.50 & 3.1 & 3.32 \\
\hline & $d$ & 1.57 & 1.52 & 2.86 & 3.32 \\
\hline & $e$ & 1.96 & 2.23 & 3.43 & 1.72 \\
\hline & $f$ & 0.08 & 0.23 & 3.39 & 41.08 \\
\hline & $\mathrm{TS}_{a-b}$ & 3.64 & 4.66 & - & - \\
\hline & $\mathrm{TS}_{a-d}^{a-b}$ & 7.01 & 6.73 & - & - \\
\hline & $\mathrm{TS}_{a-f}$ & 6.55 & 7.60 & - & - \\
\hline & $\mathrm{TS}_{a-e}$ & 6.13 & 7.01 & - & - \\
\hline & $\mathrm{TS}_{b-c}$ & 5.32 & 6.17 & - & - \\
\hline & $\mathrm{TS}_{c-f}$ & 6.96 & 7.66 & - & - \\
\hline & $\mathrm{TS}_{c-e}$ & 7.07 & 6.76 & - & - \\
\hline & $\mathrm{TS}_{d-f}$ & 6.55 & 7.66 & - & - \\
\hline & $\mathrm{TS}_{d-e}$ & 6.94 & 6.45 & - & - \\
\hline \multirow[t]{15}{*}{ Solution } & $a$ & 0.03 & 0.03 & 3.62 & 40.20 \\
\hline & $b$ & -0.28 & 1.28 & 2.83 & 4.89 \\
\hline & $c$ & 0.17 & 1.78 & 3.51 & 2.10 \\
\hline & $d$ & 0.14 & 2.07 & 2.68 & 1.29 \\
\hline & $e$ & 0.09 & 2.03 & 3.33 & 1.36 \\
\hline & $f$ & -0.13 & -0.10 & 3.92 & 50.17 \\
\hline & $\mathrm{TS}_{a-b}$ & -0.52 & 2.59 & - & - \\
\hline & $\mathrm{TS}_{a-d}$ & -0.05 & 6.91 & - & - \\
\hline & $\mathrm{TS}_{a-f}$ & -0.44 & 4.45 & - & - \\
\hline & $\mathrm{TS}_{a-e}$ & -0.84 & 4.45 & - & - \\
\hline & $\mathrm{TS}_{b-c}^{a-c}$ & 4.98 & 11.49 & - & - \\
\hline & $\mathrm{TS}_{c-f}$ & 0.40 & 7.35 & - & - \\
\hline & $\mathrm{TS}_{c-e}$ & 3.90 & 9.98 & - & - \\
\hline & $\mathrm{TS}_{d-f}$ & 0.15 & 7.37 & - & - \\
\hline & $\mathrm{TS}_{d-e}$ & -0.49 & 5.97 & - & - \\
\hline
\end{tabular}




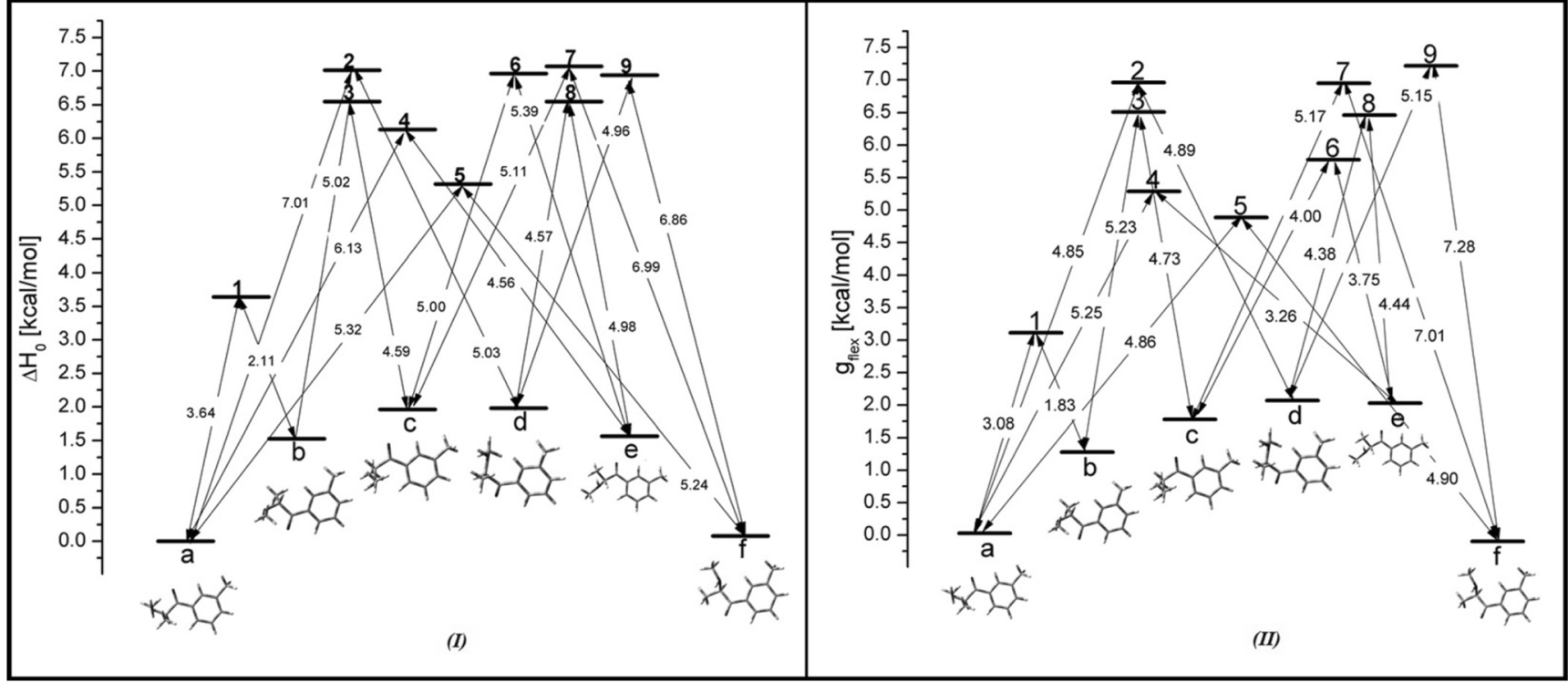

Fig. 3 Energy profile in gas (a) and solution (b) for rotational transitions calculated on MP2 level of theory with 6-31G(d,p) basis set for 3-MMC

different low-energy conformers connected through transition states. All computed data have been represented in Table 1 for gas phase, which suggested that the difference between the lowest- and the highest-energy conformer is $1.96 \mathrm{kcal} / \mathrm{mol}$ for MP2 (conformer $e$ ). The highest barrier for rotation between two conformers is $7.07 \mathrm{kcal} / \mathrm{mol}$ (rotation $c \leftrightarrow e$ ). The<smiles>CNC(C)C(=O)c1cccc(C(=O)O)c1</smiles>

Fig. 4 Main probable metabolites of 3-MMC and 4-MMC [26] 


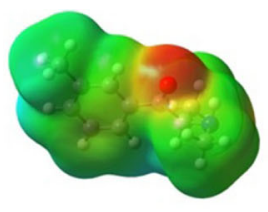

a

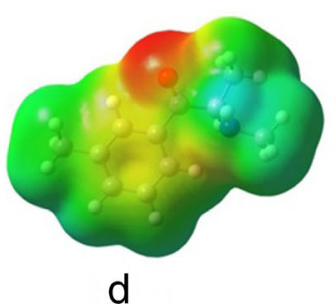

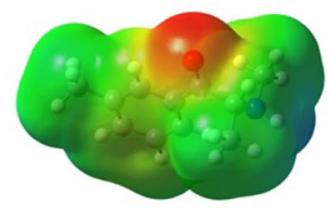

b

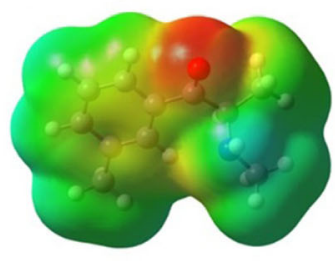

e

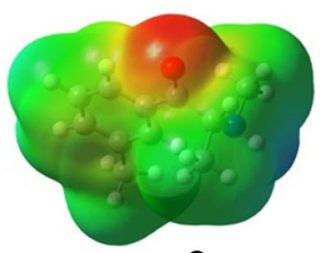

C

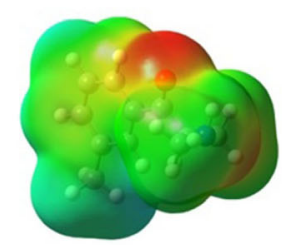

$f$

Fig. 5 Molecular electrostatic potential surfaces (MEPSs) of all rotational conformers of 3-MMC. Order of increasing electron density is blue $<$ green $<$ yellow $<$ orange $<$ red

lowest rotational barrier is $3.64 \mathrm{kcal} / \mathrm{mol}$ for transition $a \leftrightarrow b$. The most populated conformers are $a$ and $f$, and they do not change with the change of different level of theory. Transition barrier between conformers $a$ and $f$ is equal to $6.55 \mathrm{kcal} / \mathrm{mol}$. The energy profile calculated on MP2 level with $6-31 \mathrm{G}(\mathrm{d}, \mathrm{p})$ basis set for gas and solution phase is shown in Fig. 3a, b, respectively. In Fig. 3b, the most propagated conformer is conformer $f$, whose propagation changed from 41.08 to $50.17 \%$ in solution. However, conformer $a$ has changed its propagation from 47.02 to $40.20 \%$. Energy difference between highest- and lowest-energy conformer is equal to $1.98 \mathrm{kcal} / \mathrm{mol}$. The highest barrier is $7.07 \mathrm{kcal} / \mathrm{mol}$ between conformers $d$ and $f$ in gas phase and $11.49 \mathrm{kcal} / \mathrm{mol}$ between conformers $b$ and $c$. The lowest barrier in solution is $2.59 \mathrm{kcal} /$ mol for transition between conformers $a$ and $b$. Solution lowers energy difference between conformers, which is true for all conformers. The solution affects rotational barriers by raising them significantly. All calculated energies for solvent and population data have been reported in Table 1. Structural data for 3-MMC has been provided in Table S2 in supplementary materials.
In Fig. 4a, we proposed probable metabolic pathway and metabolites of 3-MMC, which is matched with the proposed pathway by Meyer and Maurer [25]. Figure 5 presents the MEPSs of all rotational conformers of 3-MMC. In all cases, the most negative surface region is around oxygen atom. Conformers $a, d$, and $f$ have negative region around nitrogen atom as well. It suggests that attacks of hydrogen go to the oxygen atom first as it is supported from the proposed metabolic pathway.

Experimental IR [11] is compared with the calculated IR spectrum of 3-MMC and presented in Fig. 6a. The shape of calculated spectrum is almost similar to calculated one. In Table 2, wave numbers of characteristic vibrations of 3$\mathrm{MMC}$ for both experimental and calculated spectrum are reported. The carbonyl $\mathrm{C}=\mathrm{O}$ stretching band for 4-MMC is approximately at $1686 \mathrm{~cm}^{-1}$ for experiment and $1738 \mathrm{~cm}^{-1}$ for calculated one. The aromatic $\mathrm{C}=\mathrm{C}$ ring vibration or ring "breathing" bands is approximately at $1603 \mathrm{~cm}^{-1}$ for experiment and $1678 \mathrm{~cm}^{-1}$ for computed one, and it is important to note that the peak is very strong for 3-MMC in both cases. The out-of-plane $\mathrm{C}-\mathrm{H}$ bending bands are in the region of $675-$
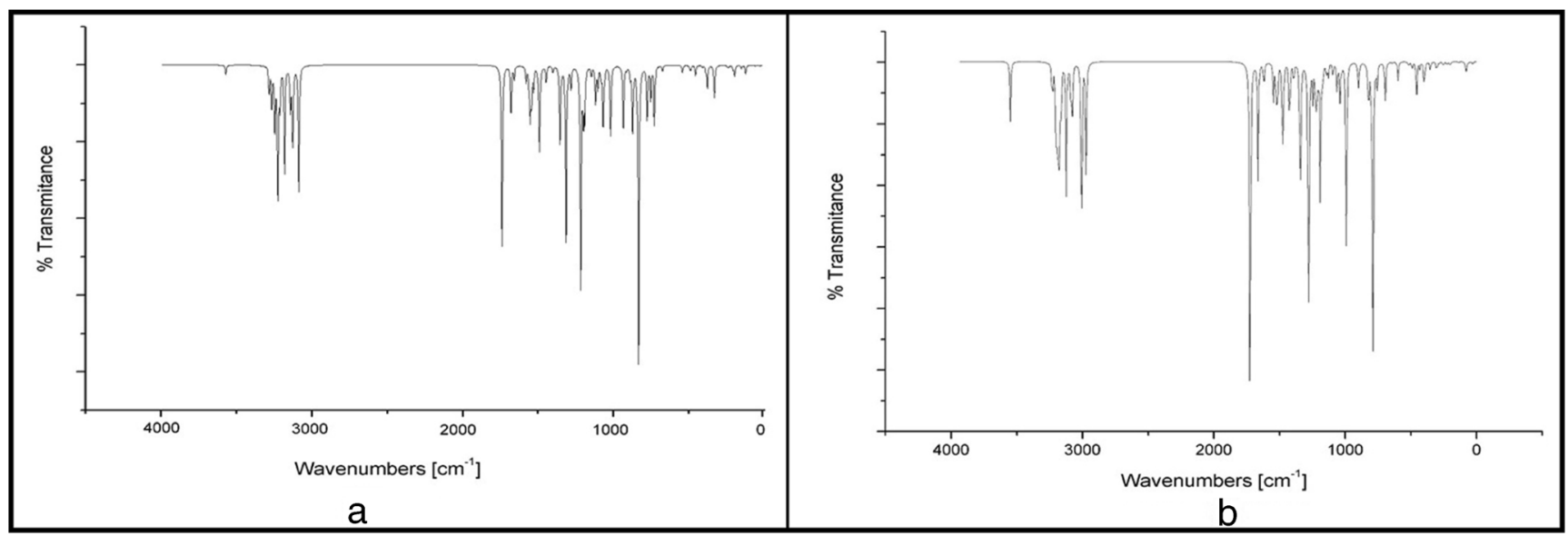

Fig. 6 Calculated IR spectrum in solution for a 3-MMC and b 4-MMC 
Table 2 Experimental and calculated characteristic vibrations of 3$\mathrm{MMC}$ and 4-MMC employing IR spectrum

\begin{tabular}{lccl}
\hline & $\begin{array}{l}\text { Experimental } \\
\left(\mathrm{cm}^{-1}\right)\end{array}$ & $\begin{array}{l}\text { Calculated } \\
\left(\mathrm{cm}^{-1}\right)\end{array}$ & Vibration \\
\hline 3-MMC & 1686 & 1738 & $\mathrm{C}=\mathrm{O}$ stretching \\
& 1603 & 1678 & $\mathrm{C}=\mathrm{C}$ ring vibration \\
& $675-894$ & $673-932$ & $\mathrm{C}-\mathrm{H}$ bending \\
& 3434 & 3569 & $\mathrm{~N}-\mathrm{H}$ stretching \\
4-MMC & 1685 & 1720 & $\mathrm{C}=\mathrm{O}$ stretching \\
& 1600 & 1672 & $\mathrm{C}=\mathrm{C}$ ring vibration \\
& $675-900$ & $695-907$ & $\mathrm{C}-\mathrm{H}$ bending \\
& 3416 & 3549 & $\mathrm{~N}-\mathrm{H}$ stretching \\
\hline
\end{tabular}

Source: [11]

$894 \mathrm{~cm}^{-1}$ for experiment and $673-932 \mathrm{~cm}^{-1}$ for calculated IR. The N-H stretching band is approximately 3569 and $3434 \mathrm{~cm}^{-1}$ for calculated and experiment IR, respectively.

The NBO analysis revealed that 3-MMC has two main resonance structures. The 3-MMC has three lonely pairs (LPs) located on nitrogen and oxygen atoms, where two of them are located on oxygen atom. Oxygen LP donate electrons to $\mathrm{C}-\mathrm{C}^{*}$ antibonding with energy 23.32 and $22.98 \mathrm{kcal} /$ mol. A double bond between the atoms $\mathrm{O}$ and $\mathrm{C}$ results from the overlap of $\mathrm{sp}^{2.30} \mathrm{~d}^{0.01}$ hybrid on $\mathrm{C}$ with $\mathrm{sp}^{1.32} \mathrm{~d}^{0.01}$ hybrid on $\mathrm{O}$ and $\mathrm{sp}$. hybrid on $\mathrm{C}$ with $\mathrm{sp}$. hybrid on $\mathrm{O}$ for the second bond. The steric properties of molecule show total steric exchange energy (SXE) of $1160.10 \mathrm{kcal} / \mathrm{mol}$ with pairwise sum of $388.40 \mathrm{kcal} / \mathrm{mol}$. The strongest interaction of SXE is $19.87 \mathrm{kcal} / \mathrm{mol}$ for O-LP/C-C. The overlapping orbital has been shown in Fig. 7. Total dipole moment is equal to 3.31 D, where Lewis and non-Lewis contributions to the dipole moment are 3.54 and $1.17 \mathrm{D}$, respectively.

\section{4-MMC}

The 4-MMC (Fig. 1) has three low-energy conformers (Fig. 8) connected through three transition states. Figure $9 \mathrm{a}, \mathrm{b}$ shows the energetic profile for gas phase and solution calculated on MP2 level using 6-31G(d,p) basis set. The highest conformer lays about $1.02 \mathrm{kcal} /$ mol above the lowest-energy conformer. The highest barrier is between conformer 2 and 3, and it is $11.42 \mathrm{kcal} /$ mol above the lowest-energy conformer. The most populated conformer is conformer 1 with $48.26 \%$, and the second most populated energy conformer is conformer 3 .

All computed thermodynamic data for 4-MMC in gas phase and in solvent have been represented in Table 3 . In solution, the lowest-energy conformer is, like in gas phase, conformer 1. Shape of energy profile changes in solvent, and energies of conformers 2 and 3 have been also changed in solvent phase. Therefore, conformer 3 becomes the highest-energy one in solvent phase. The most populated energy conformer is conformer 1 with $88.90 \%$ of population. Population of conformer 3 has decreased to $4.67 \%$ in solution. Dipole moment in solution, as it has been expected, has increased in solution (Table 3). Structural data for 4-MMC has been provided in Table S2 in supplementary materials.

Experimental IR [11] is compared with the calculated IR spectrum of 4-MMC and presented in Fig. 6b. Most interestingly, the shape of calculated spectrum is almost similar to the experimental one. In Table 2, wave numbers of characteristic vibrations of 4-MMC for both experimental and calculated spectrum are reported. The carbonyl $\mathrm{C}=\mathrm{O}$ stretching band for 4-MMC is approximately at $1685 \mathrm{~cm}^{-1}$ for experiment and $1720 \mathrm{~cm}^{-1}$ for calculated one. The aromatic $\mathrm{C}=\mathrm{C}$ ring vibration or ring "breathing" bands is approximately at $1600 \mathrm{~cm}^{-1}$ for experiment and $1672 \mathrm{~cm}^{-1}$ for computed one, and it is important to note that the peak is very strong for 4MMC in both cases. The out-of-plane $\mathrm{C}-\mathrm{H}$ bending bands are in the region of $675-900 \mathrm{~cm}^{-1}$ for experiment and $695-907 \mathrm{~cm}^{-1}$ for calculated IR. In experimental IR, spectra are visible in overtone bands (1700$2000 \mathrm{~cm}^{-1}$ ), which have not been calculated in theoretical one. The $\mathrm{N}-\mathrm{H}$ stretching band is approximately 3549 and $3416 \mathrm{~cm}^{-1}$ for calculated and experiment IR, respectively.

Para-mephedrone (4-MMC) has highly delocalized structure with two most important resonance structures. The 4MMC molecule has three LPs, where two LPs localized on oxygen and one LP localized on nitrogen atoms. Total SXE
Fig. 7 Overlapping orbitals of a 3-MMC and b 4-MMC, where light bluelyellow is the lonely pair of oxygen atom and dark bluel yellow is the $\mathrm{C}-\mathrm{C}$ binding

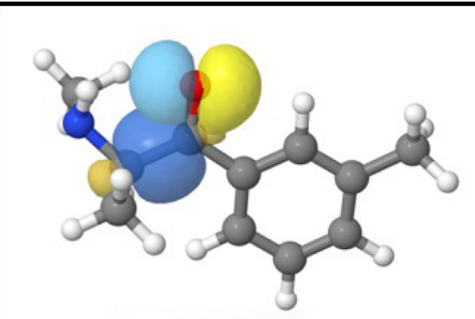

a 3-MMC

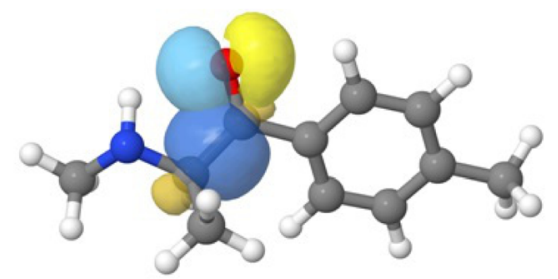

b 4-MMC 
Fig. 8 All three conformers of 4MMC calculated with 6-31G(d,p) basis set on MP2 level
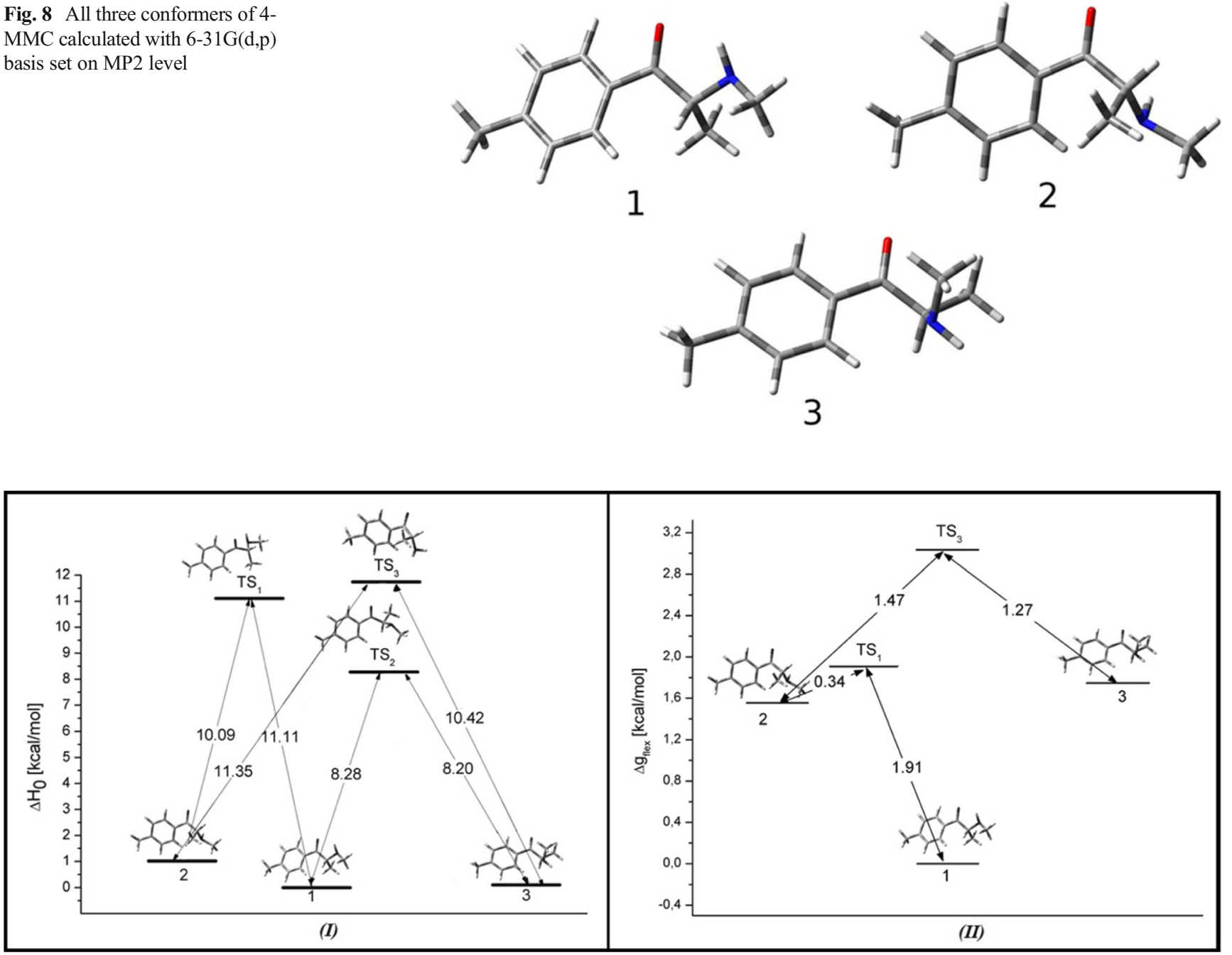

Fig. 9 Energy profile for 4-MMC calculated at MP2 level with 6-31G(d,p) basis set in a gas phase and $\mathbf{b}$ solution

for 4-MMC is equal to $1160.52 \mathrm{kcal} / \mathrm{mol}$, where pairwise sum is only $391.00 \mathrm{kcal} / \mathrm{mol}$. A double bond between the atoms $\mathrm{O}$ and $C$ results from the overlap of a $\mathrm{sp}^{2.29} \mathrm{~d}^{0.01}$ hybrid on $\mathrm{C}$ with a $\mathrm{sp}^{1.34} \mathrm{~d}^{0.01}$ hybrid on $\mathrm{O}$ and a $\mathrm{sp}$. hybrid on $\mathrm{C}$ with a $\mathrm{sp}$. hybrid on $\mathrm{O}$ for the other bond. The overlapping orbitals have been shown in Fig. 7. The highest overlapping energy is between lonely pair localized on oxygen atom, and $\mathrm{C}-\mathrm{C}$ bond is equal to $19.14 \mathrm{kcal} / \mathrm{mol}$.

Analysis of MEPS (Fig. 10) of 4-MMC shows that the most reactive site of mephedrone moiety is present in part of nitrogen and oxygen atoms, and the most reactive one is molecule number 1 (Fig. 10), which is the lowest-energy conformer and specially visible in proposed metabolites of 4-MMC in Fig. 4b. In one metabolism reaction path, $\mathrm{H}^{+}$ion attacks nitrogen atom and breaks bond between $\mathrm{CH}_{4}$ group and moiety resulting release of $\mathrm{CH}_{4}$ [25]. Calculation shows that for this reaction, enzyme is necessary to decrease barrier, which is $142 \mathrm{kcal} / \mathrm{mol}$ high for this reaction. Experiment shows that mephedrone reacts with dopamine and serotonine [26]. Dopamine is one of the neurotransmitters, a chemical messenger heavily active in the mesolimbic and mesocortical reward pathways. As discussed in the "Introduction" section,

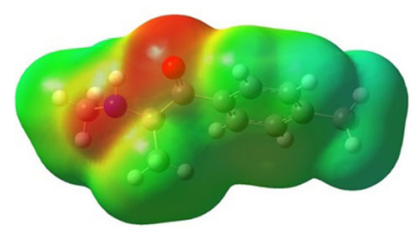

1

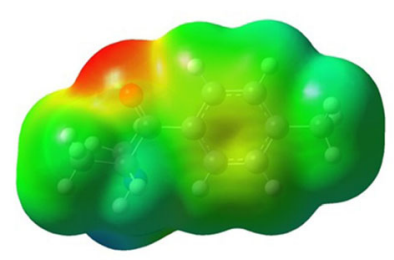

3

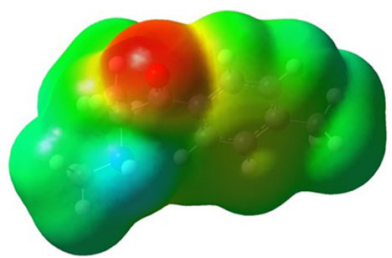

2

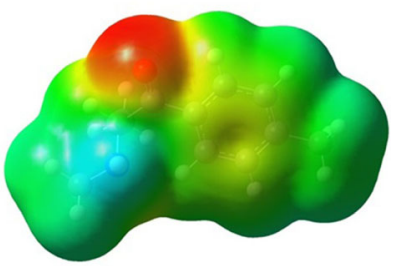

4
Fig. 10 Molecular electropotential surfaces of all conformers of 4-MMC calculated with $6-31 \mathrm{G}(\mathrm{d}, \mathrm{p})$ basis set on MP2 level. Order of increasing electron density is blue $<$ green $<$ yellow $<$ orange $<$ red 
Table 3 Thermodynamic data of low-energy conformers of 4$\mathrm{MMC}$ in $\mathrm{kcal} / \mathrm{mol}$ in gas and solution phase calculated at MP2 level

\begin{tabular}{cccccc}
\hline Phase & Conformer & $\Delta H_{0}(\mathrm{kcal} / \mathrm{mol})$ & $\Delta G(\mathrm{kcal} / \mathrm{mol})$ & Dipole moment $(\mathrm{D})$ & Population $(\%)$ \\
\hline \multirow{2}{*}{ Gas } & 1 & 0.00 & 0.32 & 3.92 & 48.26 \\
& 2 & 1.02 & 1.15 & 2.89 & 8.60 \\
& 3 & 0.07 & 0.00 & 2.88 & - \\
& $\mathrm{TS}_{1}$ & 11.12 & 7.72 & - & - \\
& $\mathrm{TS}_{2}$ & 8.28 & 4.43 & - & - \\
& $\mathrm{TS}_{3}$ & 11.42 & 8.15 & 5.24 & 88.90 \\
\multirow{5}{*}{ Solution } & 1 & 0.00 & 0.00 & 3.72 & 4.67 \\
& 2 & 0.16 & 1.56 & 3.89 & - \\
& 3 & 1.38 & 1.75 & - & - \\
& $\mathrm{TS}_{1}$ & -4.13 & 1.91 & - & - \\
\hline
\end{tabular}

cathinone and amphetamine target various neurotransmitters involved in various pathways of the central nervous system functions [27, 28]. According to our quantum-mechanical study, we believe that mephedrone acts the same way as amphetamine and cathinone and further introspection is required, which is under investigation.

\section{Conclusion}

In this work, we carried out conformational analysis of isomers of mephedrone derivatives at the MP2/6-31G(d,p) level. The dependence of the low-energy conformers of mephedrone derivatives on aqueous solvation was studied using the MP2/ COSMO model, and the MEP surfaces were generated for these low-energy surfaces to interpret the nature of practical reaction regions. Our calculations indicate that 3-MMC and 4MMC have several low-energy conformers in the gas as well as in solvent medium. The transition states of 4-MMC lowenergy conformers are higher than the 3-MMC derivative. The number of conformers and energy barrier height could explain different influence of 3-MMC and 4-MMC in human body as low rotational barriers and numbers of conformers for 3 MMC due to molecular shape could not always fit to the neurotransmitter, and therefore, inhibition is weaker. In solution, there is no transition state between conformers 1 and 3 (4-MMC). These low-energy conformers are connected through low-barrier transition states, indicating that they can be converted from one form to the other. The calculated IR spectra for both MMCs are similar to experimental one; thus, we are sure that the calculated molecules correspond good with reality. From steric evaluation of both derivatives by NBO analysis, we find a difference in steric exchange energy of $0.42 \mathrm{kcal} / \mathrm{mol}$ and in the sum of pairwise contributions of $2.60 \mathrm{kcal} / \mathrm{mol}$ between 3-MMC and 4-MMC. Both values indicate that steric repulsions are favoring the 4-MMC. The MEP surfaces revealed potential regions of 3- and 4-MMC, where reaction can take place in the body. This study also reveals the importance of low-energy conformers of mephedrone derivatives in exhibiting its specific biological properties. Understanding the structure offers a starting point for defining the mechanism of action and provides for the development of antidotes for drug poisoning, overdose, or addiction.

Acknowledgements The authors would like to thank the Wroclaw Supercomputing and Networking Center (WCSS) for the generous allotment of computer time. We would also like to thank the Mississippi Center for Supercomputing Research (MCSR) for providing the computing time. Authors want to thank the National Science Foundation (NSF/ CREST HRD-1547754) for financial support.

\section{Compliance with ethical standards}

Conflict of interest The authors declare that they have no conflict of interest.

Open Access This article is distributed under the terms of the Creative Commons Attribution 4.0 International License (http:// creativecommons.org/licenses/by/4.0/), which permits unrestricted use, distribution, and reproduction in any medium, provided you give appropriate credit to the original author(s) and the source, provide a link to the Creative Commons license, and indicate if changes were made.

\section{References}

1. Baumann MH, Ayestas MA, Partilla JS, et al. (2011) The designer methcathinone analogs, mephedrone and methylone, are substrates for monoamine transporters in brain tissue. Neuropsychopharmacology 37:1192-1203. doi:10.1038/npp.2011.304

2. Gregg RA, Rawls SM (2014) Behavioral pharmacology of designer cathinones: a review of the preclinical literature. Life Sci 97:27-30. doi:10.1016/j.1fs.2013.10.033

3. Valente MJ, Guedes de Pinho P, de Lourdes BM, et al. (2014) Khat and synthetic cathinones: a review. Arch Toxicol 88:15-45. doi: 10.1007/s00204-013-1163-9

4. Green AR, King MV, Shortall SE, Fone KCF (2014) The preclinical pharmacology of mephedrone; not just MDMA by another name. Br J Pharmacol 171:2251-2268. doi:10.1111/bph.12628

5. Bonano JS, Glennon RA, De Felice LJ, et al. (2014) Abuse-related and abuse-limiting effects of methcathinone and the synthetic "bath salts" cathinone analogs methylenedioxypyrovalerone (MDPV), 
methylone and mephedrone on intracranial self-stimulation in rats. Psychopharmacology 231:199-207. doi:10.1007/s00213-0133223-5

6. de Buranga Sanchez MJS (1929) Bull Soc Chim Fr 45:284-286

7. Bonano JS, Banks ML, Kolanos R, et al. (2015) Quantitative structure-activity relationship analysis of the pharmacology of para-substituted methcathinone analogues. Br J Pharmacol 172: 2433-2444. doi:10.1111/bph. 13030

8. Sakloth F, Kolanos R, Mosier PD, et al. (2015) Steric parameters, molecular modeling and hydropathic interaction analysis of the pharmacology of para-substituted methcathinone analogues. Br J Pharmacol 172:2210-2218. doi:10.1111 /bph.13043

9. Hall C, Heyd C, Butler C, Yarema M (2014) "Bath salts" intoxication: a new recreational drug that presents with a familiar toxidrome. Can J Emerg Med 16:171-176. doi:10.2310 /8000.2013.131042

10. Gatch MB, Taylor CM, Forster MJ (2013) Locomotor stimulant and discriminative stimulus effects of "bath salt" cathinones. Behav Pharmacol 24:437-447. doi:10.1097/FBP.0b013 e328364166d

11. Power JD, McGlynn P, Clarke K, et al. (2011) The analysis of substituted cathinones. Part 1: chemical analysis of 2-, 3- and 4methylmethcathinone. Forensic Sci Int 212:6-12

12. Torrance H, Cooper G (2010) The detection of mephedrone (4methyl methcathinone) in 4 fatalities in Scotland. Forensic Sci Int 202:e62-e63. doi:10.1016/j.forsciint.2010.07.014

13. Carhart-Harris RL, King LA, Nutt DJ (2011) A web-based survey on mephedrone. Drug Alcohol Depend 2-5. doi:10.1016/ j.drugalcdep.2011.02.011

14. Kolodziejczyk W, Kar S, Hill GA, Leszczynski J (2016) A comprehensive computational analysis of cathinone and its metabolites using quantum mechanical approaches and docking studies. Struct Chem 27:1291-1302. doi:10.1007/s11224-016-0779-9
15. Møller C, Plesset MS (1943) Note on an approximation treatment for many-electron systems. Phys Rev 46:618. doi:10.1103 /PhysRev.56.841.2

16. Frisch MJ, Trucks GW, Schlegel HB, et al. (2009) Gaussian 2009

17. Barone V, Cossi M (1998) Quantum calculation of molecular energies and energy gradients in solution by a conductor solvent model. J Phys Chem A 102:1995-2001

18. Hwang J-K, King G, Creighton S, Warshel A (1988) Simulation of free energy relationships and dynamics of SN2 reactions in aqueous solution. J Am Chem Soc 110:52975311

19. Warshel A (1991) Comput Model Chem React Enzym Solut

20. Florián J, Warshel A (1998) Phosphate ester hydrolysis in aqueous solution: associative versus dissociative mechanisms. J Phys Chem B 102:719-734

21. Florián J, Štrajbl M, Warshel A (1998) Conformational flexibility of phosphate, phosphonate, and phosphorothioate methyl esters in aqueous solution. J Am Chem Soc 120:7959-7966

22. Davidson N (1962) Stat Mech

23. Glendening ED, Badenhoop JK, Reed AE, et al. (2013) NBO 6.0

24. Tobergte DR, Curtis S (2013) Discovering chemistry with natural bond orbitals. J Chem Inf Model. doi:10.1017/CBO9781107415324.004

25. Meyer MR, Maurer HH (2010) Metabolism of designer drugs of abuse: an updated review. Curr Drug Metab 11:468-482

26. Martínez-Clemente J, Escubedo E, Pubill D, Camarasa J (2011) Interaction of mephedrone with dopamine and serotonin targets in rats. Eur Neuropsychopharmacol. doi:10.1016/j. euroneuro.2011.07.009

27. Moore KE (1977) The actions of amphetamine on neurotransmitters: a brief review. Biol Psychiatry 12:451-462

28. Kalix P (1985) The khat alkaloid (-)cathinone acts like amphetamine on physiological catecholamine stores. NIDA Res Monogr Ser NO 55:286 